\title{
Differential Effect of Calsequestrin Ablation on Structure and Function of Fast and Slow Skeletal Muscle Fibers
}

\author{
Cecilia Paolini, ${ }^{1}$ Marco Quarta, ${ }^{2,3}$ Laura D’Onofrio, ${ }^{1}$ Carlo Reggiani, ${ }^{2,4}$ \\ and Feliciano Protasi ${ }^{1}$ \\ ${ }^{1}$ CeSI-Center for Research on Ageing \& DNI-Department of Neuroscience and Imaging, \\ Università Gabriele d'Annunzio of Chieti, 66100 Chieti, Italy \\ ${ }^{2}$ Department of Anatomy and Physiology, University of Padova, 35131 Padova, Italy \\ ${ }^{3}$ Department of Neurology and Neurological Sciences, Stanford University, Palo Alto, CA 94304, USA \\ ${ }^{4}$ CNR Institute of Neurosciences, 56724 Pisa, Italy
}

Correspondence should be addressed to Carlo Reggiani, carlo.reggiani@unipd.it

Received 30 April 2011; Accepted 12 July 2011

Academic Editor: Lars Larsson

Copyright (C) 2011 Cecilia Paolini et al. This is an open access article distributed under the Creative Commons Attribution License, which permits unrestricted use, distribution, and reproduction in any medium, provided the original work is properly cited.

\begin{abstract}
We compared structure and function of EDL and Soleus muscles in adult (4-6 m) mice lacking both Calsequestrin (CASQ) isoforms, the main SR $\mathrm{Ca}^{2+}$-binding proteins. Lack of CASQ induced ultrastructural alterations in $\sim 30 \%$ of Soleus fibers, but not in EDL. Twitch time parameters were prolonged in both muscles, although tension was not reduced. However, when stimulated for $2 \mathrm{sec}$ at $100 \mathrm{hz}$, Soleus was able to sustain contraction, while in EDL active tension declined by 70-80\%. The results presented in this paper unmask a differential effect of CASQ1\&2 ablation in fast versus slow fibers. CASQ is essential in EDL to provide large amount of $\mathrm{Ca}^{2+}$ released from the SR during tetanic stimulation. In contrast, Soleus deals much better with lack of CASQ because slow fibers require lower $\mathrm{Ca}^{2+}$ amounts and slower cycling to function properly. Nevertheless, Soleus suffers more severe structural damage, possibly because SR $\mathrm{Ca}^{2+}$ leak is more pronounced.
\end{abstract}

\section{Introduction}

Skeletal muscles are composed of a variety of fibers which are traditionally classified as fast and slow depending on their contractile parameters, such as time to peak in the isometric twitch or maximum shortening velocity, or as glycolytic and oxidative depending on their metabolic properties [1]. The current nomenclature, based on myosin heavy chain (MHC) isoform composition, includes 4 major fiber types, called type 1 or slow and type $2 \mathrm{~A}, 2 \mathrm{X}$, and $2 \mathrm{~B}$ or fast, respectively, each of them with specific contractile properties [2].

The differences between fast and slow fibers, however, are not restricted only to myofibrillar proteins (myosin isoforms) and to metabolic enzymes (predominance of glycolitic versus oxidative activities), but also involves other subcellular systems [3]. Importantly the kinetics of $\mathrm{Ca}^{2+}$ mobilization are profoundly different in slow versus fast twitch fibers [4-7]. Intracellular $\mathrm{Ca}^{2+}$ concentrations $\left(\left[\mathrm{Ca}^{2+}\right]_{\mathrm{i}}\right)$ and $\mathrm{Ca}^{2+}$ release/reuptake from intracellular stores (i.e., the sarcoplasmic reticulum, SR) are controlled by the sarcotubular system, a highly organized system of membranes formed by the association of invaginations of the sarcolemma, i.e., the transverse (T)-tubules, with the terminal cisternae of the SR $[8,9]$. T-tubules contain voltagegated L-type $\mathrm{Ca}^{2+}$ channel (or dihydropyridine receptors, DHPRs) which are mechanically coupled to $\mathrm{Ca}^{2+}$ release channels of the SR, the ryanodine receptors type-1 (RYR1) $[10,11]$. Interaction between DHPR and RYR1 occurs at intracellular junctions called $\mathrm{Ca}^{2+}$ release units (CRUs) or triads, which mediate excitation-contraction (EC) coupling $[12,13]$. CRUs contain several other proteins beside DHPRs and RYR1s: among them Calsequestrin (CASQ), the main intraluminal $\mathrm{Ca}^{2+}$ binding protein of the SR $[14,15]$, which is located in terminal cisternae of the junctional SR in close proximity to RYRs $[16,17]$. In skeletal muscles, CASQ exists in two isoforms known as CASQ1 (or skeletal) and CASQ2 (or cardiac). Both isoforms can be found in slow fibers, whereas only CASQ1 is expressed in fast 
fibers $[18,19]$. It has been reported that the total CASQ content is greater in fast than in slow fibers [20]. A recent quantitative analysis on single fibers from rat points to a concentration of $36 \mu \mathrm{M}$ in fast fibers (only CASQ1) versus $10 \mu \mathrm{M}$ in slow fibers (CASQ1 and CASQ2) [21]. Owing to its properties (medium-low affinity, but high capacity), CASQ provides a large SR pool of releasable $\mathrm{Ca}^{2+}$, while maintaining SR intraluminal concentrations of free $\mathrm{Ca}^{2+}$ low enough to facilitate the work of sarcoendoplasmic reticulum $\mathrm{Ca}^{2+}$ ATP-ase (SERCA) pumps. CASQ in skeletal muscle fibers plays an important dual role: (i) to buffer $\mathrm{Ca}^{2+}$ inside the SR thanks to the large number of acidic residues which allows each CASQ molecule to bind up to 60-80 $\mathrm{Ca}^{2+}$ ions [22]; (ii) to modulate $\mathrm{Ca}^{2+}$ release from the $S R$ via a tradin/junctin-mediated interaction with RYR1 [23-25]. There is evidence that CASQ1 has different polymerization rate and $\mathrm{Ca}^{2+}$ buffering properties than CASQ2 [22], suggesting the possibility that RYR1 is modulated differently by CASQ1 and 2 in fast- and slowtwitch fibers [26]. Murphy and colleagues [21] recently suggested that CASQ2 is more efficient than CASQ1 in reducing $\mathrm{SR} \mathrm{Ca}^{2+}$ leak, a property also shown in cardiac muscle [27].

Mice lacking CASQ1 were viable and fertile [28]. Nevertheless, lack of CASQ1 induced significant functional and structural modifications in skeletal fibers: CASQ1 ablation reduced dramatically the total $\mathrm{SR} \mathrm{Ca}^{2+}$ content (of about 70\%) in fast twitch from flexor digitorum brevis (FDB) muscles. However, $\mathrm{Ca}^{2+}$ transients evoked by a single stimulus was surprisingly not dramatically reduced so that twitch peak force was preserved [28]. This apparent minor functional impairment can be in part explained by morphological adaptations taking place in fast fibers, that is, profound remodelling of CRUs which forms multiple layers of junctional SR and T-tubules bearing an approximately doubled number of RYRs [28].

Both functional and structural changes were more evident in extensor digitorum longus (EDL) and in FDB, containing predominantly fast twitch fibers, than in Soleus muscle, a predominantly slow twitch muscle. There are reasons to believe that the impact of CASQ1 ablation is more evident in EDL and in FDB than in Soleus, because CASQ2 is expressed in CASQ1-null mice and quite abundant in slow twitch fibers. To explain the differential impact of CASQ1 ablation in fast versus slow fibers, we may also have to consider important functional differences: in fast fibers greater amounts of $\mathrm{Ca}^{2+}$ are released after each action potential [5], SR volume is greater, and SR is filled only to $35 \%$ of its maximal capacity [21]. In contrast SR of slow fibers is filled to its maximal capacity [21]. In view of the possible diversity in $\mathrm{Ca}^{2+}$ handling between slow and fast muscles and to investigate how complete ablation of CASQ will affect the different muscle types we studied EDL and Soleus muscle in mice lacking both CASQ isoforms [29, 30], generated by cross-breeding preexisting CASQ1-null and CASQ2-null mice [28, 31].

Interestingly, our results show that EDL and Soleus are differently affected by complete ablation of CASQ, as EDL, but not Soleus, becomes unable to maintain tension during prolonged tetanic contractions, while Soleus, but not EDL, displays the early onset of a myopathic phenotype.

\section{Materials and Methods}

2.1. CASQ-Null Mice. CASQ1-null and CASQ2-null mice were generated as previously described [28, 31]. Double (d)CASQ-null mice lacking both CASQ isoforms were generated by cross-breeding the preexisting CASQ1-null and CASQ2-null mice. C57BL/6J mice were used as wild-type (WT) controls and obtained from Charles River Italia. Mice were housed in microisolator cages, temperature $22^{\circ} \mathrm{C}, 12 \mathrm{hr}$ light/dark cycle, with free access to water and food. Mice were killed by an overdose of the anaesthetic ethylic ether, and their muscles were rapidly dissected. All experiments were conducted according to the National Institutes of Health Guide for the Care and Use of Laboratory Animals and were approved by the ethical committee of the University of Chieti and of the Department of Anatomy and Physiology, University of Padova.

\subsection{Preparation of Homogenates, Electrophoresis, Western Blot} Analysis (Figure 1). Preparation of total homogenates from WT, CASQ1-null and dCASQ-null muscles (hind limb, EDL, and Soleus), and western blot analysis were performed as previously described in 4-6 -month- old mice [28]. The antibody used was a rabbit polyclonal antibody reactive with both isoforms of CASQ (Affinity Bioreagents, USA).

2.3. Spontaneous Mortality Rate (Figure 2). The rate of spontaneous mortality under standard housing conditions was assessed during the entire life span using the KaplanMeier method in a subpopulation of mice which were not utilized for other experiments. Age- and sex-dependent probability of survival is shown in Figure 2.

2.4. Grip Strength Test (Figure 3). Strength developed by WT, CASQ1-null, and dCASQ-null male mice of 6 months of age was measured during instinctive grasp with a gripstrength-test protocol [33]. The mouse was held by the tail in proximity to a trapeze bar connected with the shaft of a Shimpo Fgv 0.5x force transducer (Metrotec Group, San Sebastián Spain). Once the mouse had firmly grabbed the trapeze, a gentle pull was exerted on the tail. The measurement of the peak force generated by each mouse with fore and hind limbs was repeated three times with appropriate intervals to avoid fatigue, and the average of the highest peak force values was normalized to the body mass [33].

2.5. Preparation and Analysis of Samples by Light and Electron Microscopy (EM) (Figure 4 and Table 1). EDL and Soleus muscles were carefully dissected from WT, CASQ1-null, and dCASQ-null at 4-6 months of age. Muscles were fixed at RT in $3.5 \%$ glutaraldehyde in $0.1 \mathrm{M}$ sodium cacodylate buffer, $\mathrm{pH} 7.2$ for $2 \mathrm{~h}$ and kept in fixative before further use. Small bundles of fixed fibers were postfixed in $2 \% \mathrm{OsO}_{4}$ in $0.1 \mathrm{M}$ sodium cacodylate buffer for $2 \mathrm{~h}$ and block stained 


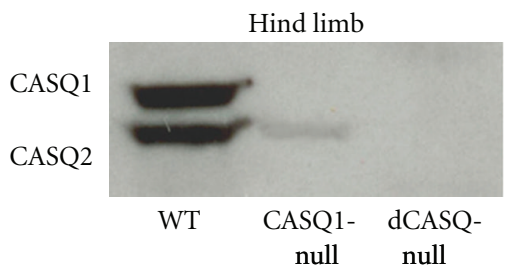

(a)

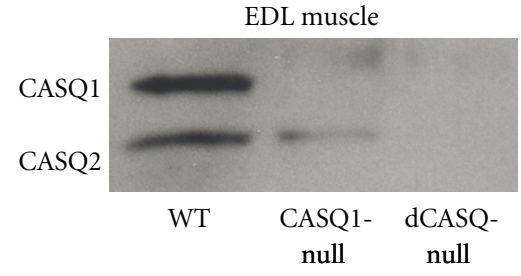

(b)

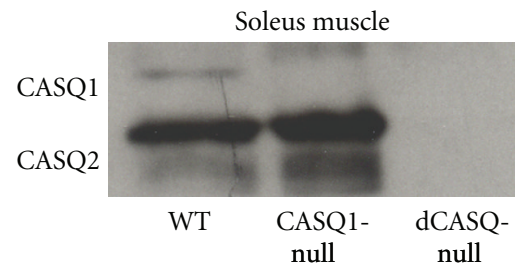

(c)

FIGURE 1: dCASQ-null mice did not express any of the two CASQ isoforms. Western blot analysis of total homogenates prepared from hind limb (a), EDL, and Soleus (b and c) muscles showed that (i) in CASQ1-null muscles CASQ1 was missing, whereas CASQ2 was still present, more in Soleus (slow twitch) and less in EDL (fast twitch); (ii) in dCASQ-null muscles both isoforms were absent.

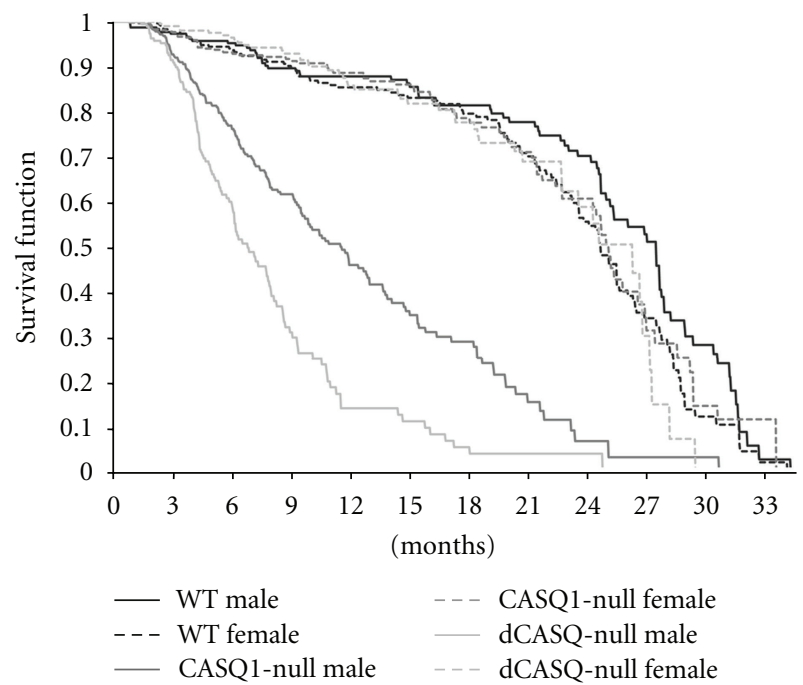

FIGURE 2: dCASQ-null male mice displayed a rate of spontaneous mortality even higher than that of CASQ1-null mice (see also [32]). Age-dependent survival analysis of male and female WT, CASQ1null, and dCASQ-null mice housed under standard conditions evaluated using the Kaplan-Meier method. Number of animals included in the study: WT: male $n=321$, female $n=318$; CASQ1null: male $n=649$, female $n=632$; dCASQ-null: male $n=200$, female $n=222$. As shown in Dainese et al. [32] male CASQ1-null mice were affected by a significantly increased rate of spontaneous mortality, particularly after 3 months of age: the additional ablation of CASQ2 worsened the phenotype.

in aqueous saturated uranyl acetate. After dehydration, specimens were embedded in an epoxy resin (Epon 812). For histological analysis, longitudinal and cross-oriented semithin sections $(300 \mathrm{~nm})$ were cut with a Leica Ultracut $\mathrm{R}$ microtome (Leica Microsystem, Vienna, Austria) using a Diatome diamond knife (DiatomeLtd. CH-2501 Biel, Switzerland). After staining with Toluidine Blue dye, the sections were viewed on a Leica DMLB fluorescence microscope (Leica Microsystem, Vienna, Austria). Quantitative analysis of damaged fibers (data in Table 1) was performed on histology images. For EM, ultrathin sections ( $35 \mathrm{~nm}$ ) were cut and, after staining in $4 \%$ uranyl acetate and lead citrate, examined with a Morgagni Series 268D electron microscope (FEI Company, Brno, Czech Republic), equipped with Megaview III digital camera.
2.6. Force and Contraction Kinetics of Isolated Intact Muscles (Figures 5 and 6). EDL and Soleus muscles were dissected from the hind limbs of WT and knock-out male mice (4 months old) in warm oxygenated Krebs solution and mounted between a force transducer (SI-H Force Transducer World Precision Instruments, Inc., Sarasota, FL, USA) and a micromanipulator-controlled shaft in a small chamber where oxygenated Krebs solution was continuously circulated. The temperature was kept constant at $25^{\circ} \mathrm{C}$. The stimulation conditions were optimized, and muscle length was increased until force development during tetanus was maximal. The responses to a single stimulus (twitch) or to a series of stimuli at various rates producing unfused or fused tetani were recorded. Time-to-peak tension, time-to-half relaxation, time-to-base tension, and peak-tension were measured in single twitches. Tension was measured in completely fused maximal tetani of different duration $(0.5-2 \mathrm{~s})$ at the peak and just after the last stimulus.

2.7. Statistical Analysis. Data were expressed as mean \pm standard errors. For analysis of force and contraction kinetics comparison between the three groups (WT, CASQ1-null, and dCASQ-null mice) was carried out using ANOVA followed by a post hoc test (Newman-keuls test), while for weight and grip test the statistical significance was assessed using the unpaired Student's $t$-test.

\section{Results}

3.1. Phenotype of dCASQ-Null Mice. The expression of the two CASQ isoforms in WT, CASQ1-null, and dCASQ-null mice was assessed by western blots of homogenates prepared from either all hind limb muscles (Figure 1(a)) or separately from EDL and Soleus (Figures 1(b) and 1(c), resp.). Analysis showed that: (a) in CASQ1-null muscles CASQ1 is missing, whereas CASQ2 is still present, more in Soleus (slow twitch) and less in EDL (fast twitch); (b) in dCASQ-null muscles both isoforms are absent. dCASQ-null mice were viable and fertile, appeared to develop and breed normally, and did not present a clear overt phenotype. However, male mice carrying the double-null mutation displayed a greatly increased mortality rate compared to WT animals, even more pronounced than that of CASQ1-null male mice (Figure 2; see also $[32,34]$ for more detail): more than $50 \%$ of 


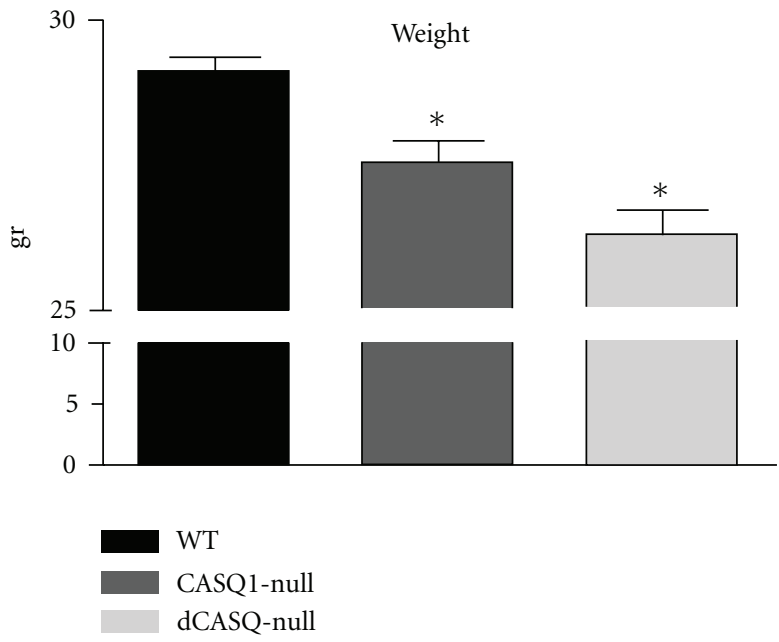

(a)

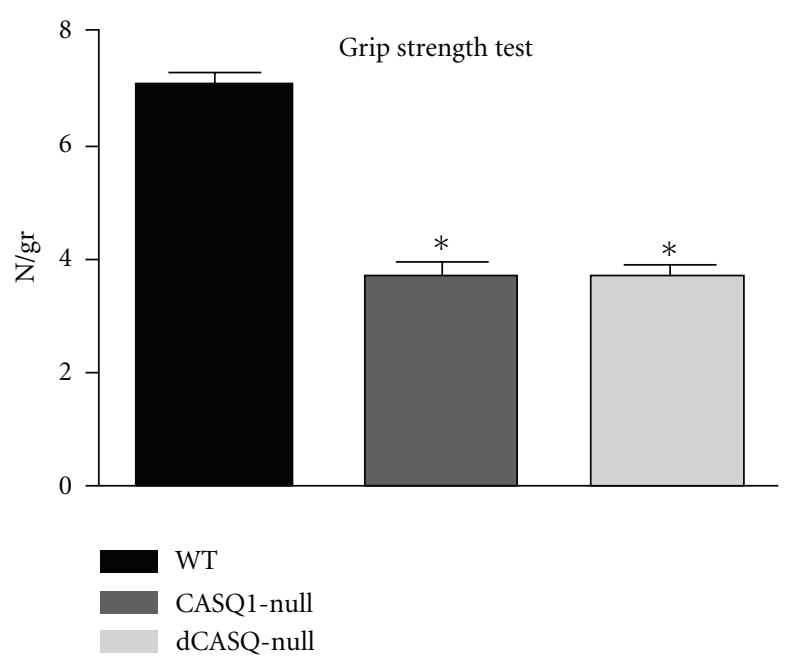

(b)

FIGURE 3: Ablation of CASQ1 and of both CASQ isoforms resulted in a significant reduction in body weight and grip strength test. (a) Ablation of CASQ1 and/or of both CASQ isoforms resulted in a significant reduction of body weight, which was more pronounced in dCASQ-null than in CASQ1-null mice ( $n=86,65$, and 33 animals for WT, CASQ1-null, and dCASQ-null, resp.). (b) The evaluation of the maximal force that mice produced while grasping a bar (grip strength test, [33]) showed that the force output of CASQ1-null and dCASQ-null mice was significantly lower than that of WT animals, but not different from each other $(n=26,39$, and 33 animals for WT, CASQ1-null, and dCASQ-null, resp.). Both body weight and grip strength test were measured in male mice. $*$ Significantly different from WT.

TABLE 1: Histological examination of adult Soleus fibers: fibers presenting structural damage were absent in WT, rare in CASQ1-null, but quite frequent in dCASQ-null. We classified abnormal fibers in two main classes: (a) fibers presenting large areas loosing cross striation, or unstructured cores, but no contractures (see Figure 4(f)); (b) fibers containing (also) contracture cores (see Figure 4(e), asterisk). (c) About $35 \%$ of Soleus fibers from dCASQ-null mice present severe structural alterations.

\begin{tabular}{|c|c|c|c|c|c|}
\hline \multirow{2}{*}{ Soleus } & \multirow{2}{*}{ Age (months) } & \multirow{2}{*}{ Total no. of fibers analyzed } & \multicolumn{2}{|c|}{ No. of fiber with alterations } & \multirow{2}{*}{ (c) Total $\%$ of altered fiber } \\
\hline & & & (a) Unstructured cores & (b) Contracture cores & \\
\hline \multirow{2}{*}{ WT } & 5 & 29 & - & - & - \\
\hline & 6 & 15 & - & - & - \\
\hline \multirow{3}{*}{ CASQ1-null } & 5 & 18 & $1(6 \%)$ & - & 6 \\
\hline & 6.2 & 27 & $1(4 \%)$ & 一 & 4 \\
\hline & & & & & Average: $5 \%$ \\
\hline \multirow{4}{*}{ dCASQ-null } & 4.8 & 25 & $7(28 \%)$ & $5(20 \%)$ & 48 \\
\hline & 4.2 & 23 & $3(13 \%)$ & $4(17 \%)$ & 30 \\
\hline & 4.8 & 47 & $2(4 \%)$ & $6(13 \%)$ & 17 \\
\hline & 4.0 & 32 & $4(13 \%)$ & $9(28 \%)$ & 41 \\
\hline
\end{tabular}

male mice died before reaching the age of 6 months. Rate of spontaneous mortality of CASQ1-null and dCASQ-null females was not significantly different from that of male and female WT (Figure 2).

\section{2. dCASQ-Null Mice Presented a Reduced Body Weight} and Produced Less Grip-Strength Force Than WT Mice. We measured the average body weight of WT, CASQ1-null, and dCASQ-null adult male mice (4-6 months of age): dCASQnull mice were on the average significantly smaller than WT and slightly smaller than CASQ1-null mice (Figure 3(a)). To assess basic neuromuscular function of living mice, we used the grip strength test, which provides a simple way to test the global muscle performance during maximal isometric contraction of short duration [33]. Figure 3(b) shows that mice lacking either CASQ1 or both CASQ isoforms developed a significantly lower force output than WT. No significant difference was detectable between mice lacking only CASQ1 and both CASQ isoforms using this method.

3.3. In dCASQ-Null Soleus Muscle 30\% of Soleus Fibers Displayed Severe Ultrastructural Damage. We performed structural analysis of EDL and Soleus muscles from adult 
WT, Soleus

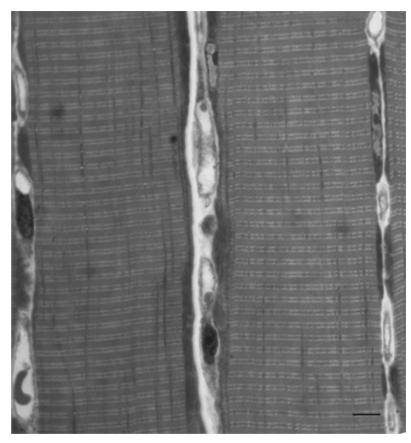

(a)

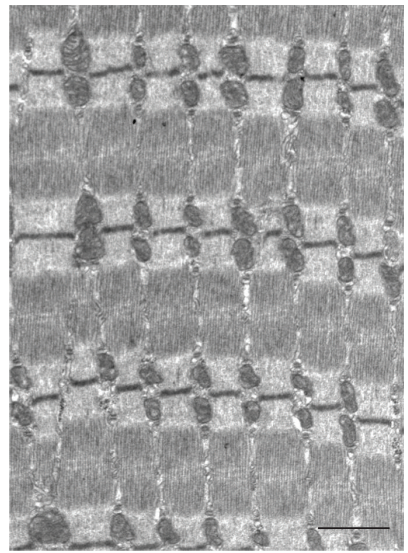

(b)
CASQ1-null, Soleus

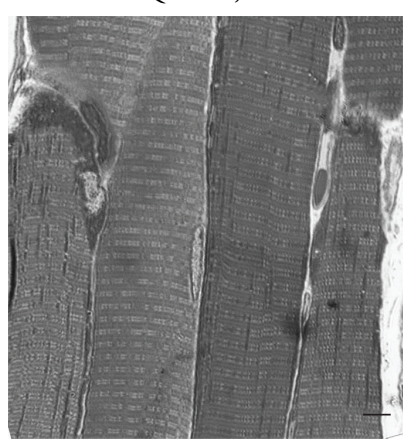

(c)

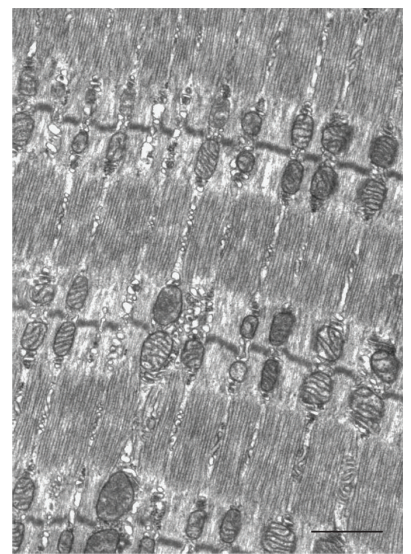

(d)

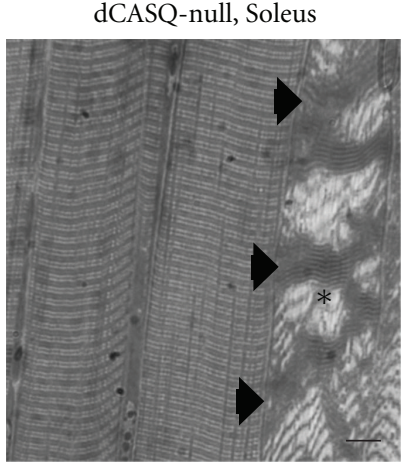

(e)

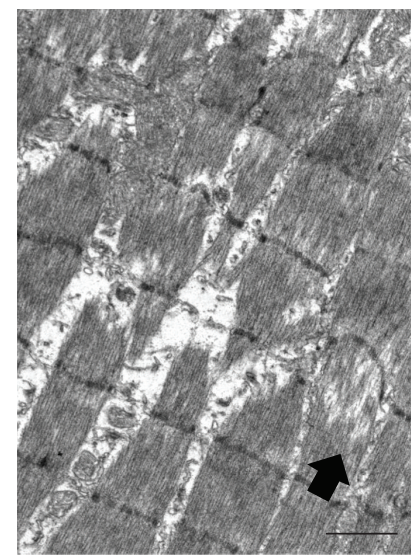

(f)

Figure 4: In dCASQ-null animals (4-6 months of age) 30\% of Soleus fibers presented severe structural damage. (a), (c), and (e): histology; (b), (d), and (f): EM. (a) and (b) WT Soleus fibers from adult mice (4-6 months of age) always showed a well-defined cross-striation and well-organized EC coupling (not shown) and mitochondrial apparatus. (c)-(f) At 4-6 months of age, 30\% of Soleus fibers (see Table 1) from dCASQ-null mice were affected by severe structural alterations, which were clearly visible both at the histological examination (e) and in EM (f). In (e) arrowheads point to three contracture cores within the same severely damaged fiber (asterisk), while in (f) is shown a small portion of a fiber presenting degeneration of the contractile elements and initial Z line streaming (arrow). Fibers with similar alterations are very rare in CASQ1-null fibers ((c) and (d)) and never seen in WT. See Table 1 for quantitative analysis.

male (4-6 months of age) WT, CASQ1-null, and dCASQnull mice using a combination of sectioning for histology and EM (Figure 4). This examination revealed that a significant percentage of fibers in dCASQ-null Soleus exhibit severe morphological alterations (Table 1). This structural damage, which was not found in EDL at this age (4-6 months), disrupts the regular cross-striation of skeletal fibers and affects large portions of the fiber interior (Figure 4(e), asterisk). Whereas structural alterations are quite variable in appearance, we classified abnormal fibers in two main classes (Table 1): (a) those presenting large areas loosing cross-striation, or unstructured cores, but no contractures (Figure 4(f)); (b) those containing also areas of contracture (Figure 4(e), asterisk). Fibers containing unstructured cores were frequent in Soleus from dCASQnull mice, but rare in CASQ1-null Soleus and totally absent in WT (Table 1, Column (a)). Fiber presenting contracture cores, on the other hand, were never found in Soleus muscles from WT and CASQ1-null mice, whereas again they were quite frequent in Soleus fibers from dCASQ-null (Table 1, Column (b)). Overall, $\sim 35 \%$ of Soleus fibers from
dCASQnull mice showed structural alterations (Table 1, Column (c)).

\subsection{Ablation of Both CASQ Isoforms Results in an Alteration of} Twitch Contractile Kinetics in Both EDL and Soleus. In order to assess the effects of the complete removal of CASQ on the contractile performance of fast- and slow-twitch muscles, we dissected EDL and Soleus muscles from WT, CASQ1-null, and dCASQ-null mice and studied their function ex vivo. The altered kinetics profile of the contractile cycle previously described in CASQ1-null muscle [28] was also evident in dCASQ-null muscles. The changes included a significant prolongation of time-to-peak tension (i.e. from the stimulus to the tension peak) in EDL compared to WT, but also to CASQ1-null (Figure 5(a)). No prolongation of time-to-peak tension was seen in Soleus (Figure 5(b)). In addition, time to base (i.e., time elapsing from stimulus to the return to base line at the end of relaxation) was significantly prolonged in both EDL and Soleus muscles of knock-out mice compared to WT. The latter effect was more pronounced in dCASQnull than in CASQ1-null mice (Figures 5(c) and 5(d)). 


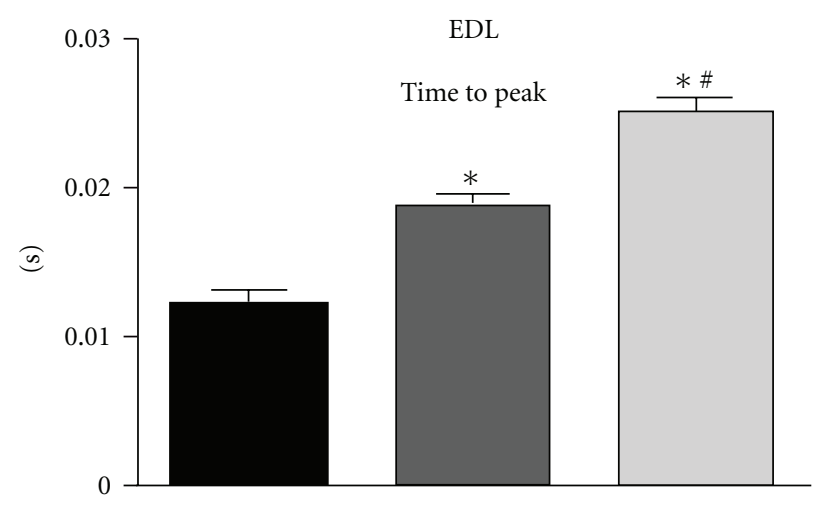

(a)

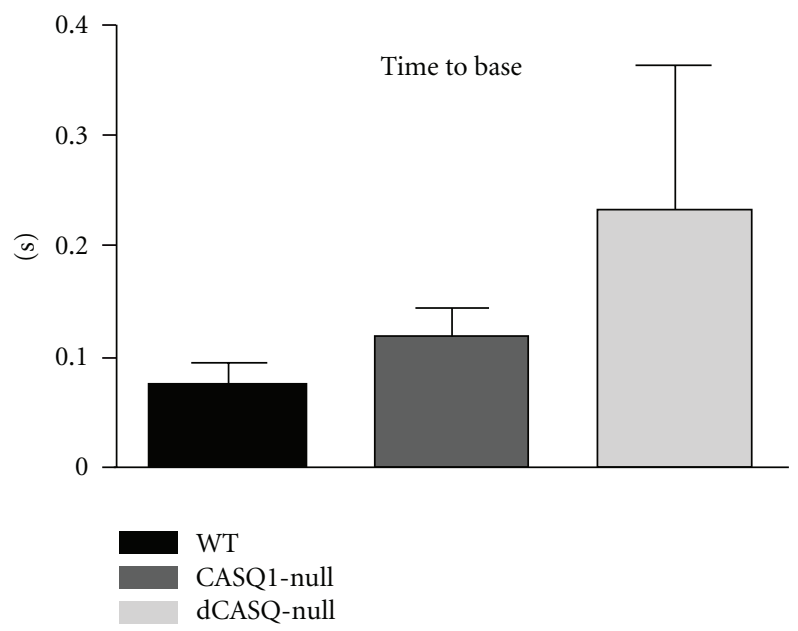

(c)

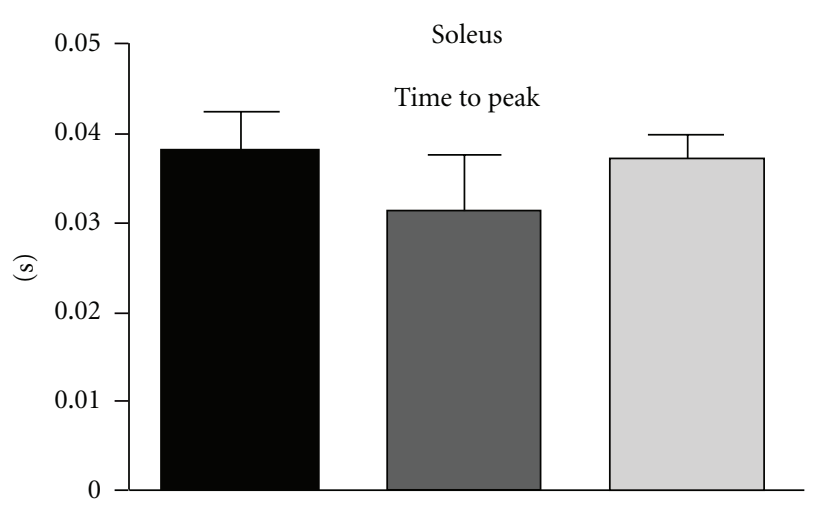

(b)

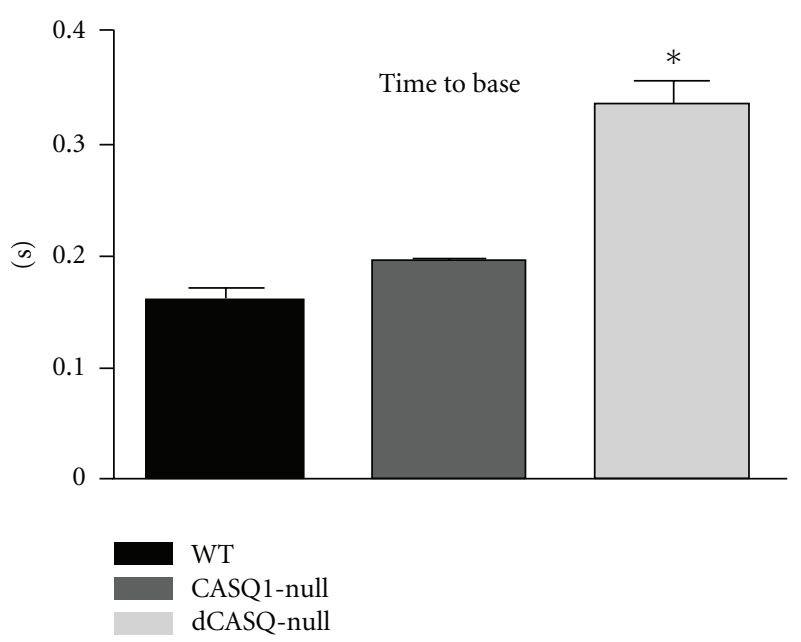

(d)

FIGURE 5: Ablation of CASQ isoforms resulted in prolongation of the twitch parameters in both EDL and Soleus. (a) and (b) Time to peak (i.e., from the stimulus to the tension peak) was prolonged in EDL from CASQ1-null, and even more in dCASQ-null, compared to WT. This effect is not seen in Soleus. (c) and (d) On the other hand, time to base (i.e., from stimulus back to base line at the end of relaxation) was prolonged compared to WT in both EDL and Soleus muscles, more in dCASQ-null than in CASQ1-null mice (EDL: WT $n=8$, CASQ1-null $n=10$, dCASQ null $n=9$, Soleus: WT $n=8$, CASQ1-null $n=10$, dCASQ null $n=10$ ). * Significantly different from WT; \#significantly different from CASQ1-null.

3.5. Ablation of CASQ Impaired the Ability of EDL, But Not of Soleus, to Maintain Tension during Tetanic Contraction. Isometric tension was determined in short fused isometric tetani with a duration just sufficient to reach peak tension (500 $\mathrm{ms}$ in EDL and $1 \mathrm{~s}$ in Soleus) and found significantly reduced in EDL, but not in Soleus muscles of CASQ1null and dCASQ-null mice (not shown). However, the most interesting result was obtained when the duration of the tetanus was prolonged up to 2 seconds (Figure 6): in both CASQ1- and dCASQ-null EDL the residual tension declined dramatically (by about 80\%) (Figures 6(a) and 6(b)), while in Soleus only a minor decline of developed tension occurred in the absence of CASQ (Figures 6(c) and 6(d)). Specifically, the tension decline of Soleus during a $2 \mathrm{~s}$ tetanus was approximately 15\% in CASQ1-null and 25\% in dCASQ-null. A careful inspection of the traces showed in Figures 6(a) and 6 (c) revealed that the kinetics of the rising phase of the isometric tetani were faster when CASQ is missing both in EDL and in Soleus. An increased early phase of $\mathrm{Ca}^{2+}$ release has been previously reported in fibers lacking CASQ isolated from dCASQ-null mice [29]. This effect can be related to the alterations of the twitch response. In agreement with the above observations, the amplitude of the twitch (measured as peak tension, not shown) was preserved, and the duration of the twitch (measured as time-to-peak and time-to-base line, Figure 5) was prolonged, thus allowing a faster tension development during repeated high frequency stimulation.

\section{Discussion}

Two isoforms, CASQ1 and CASQ2, are expressed in skeletal muscle fibers [18, 19], with CASQ1 and CASQ2 being more abundant in fast- and slow-twitch fibers, respectively [21]. The impact of their removal in different fiber types has not been investigated yet. In view of the possible diversity in $\mathrm{Ca}^{2+}$ handling between slow and fast muscles and to investigate how complete ablation of CASQ will affect the two different muscle types, we studied EDL and 


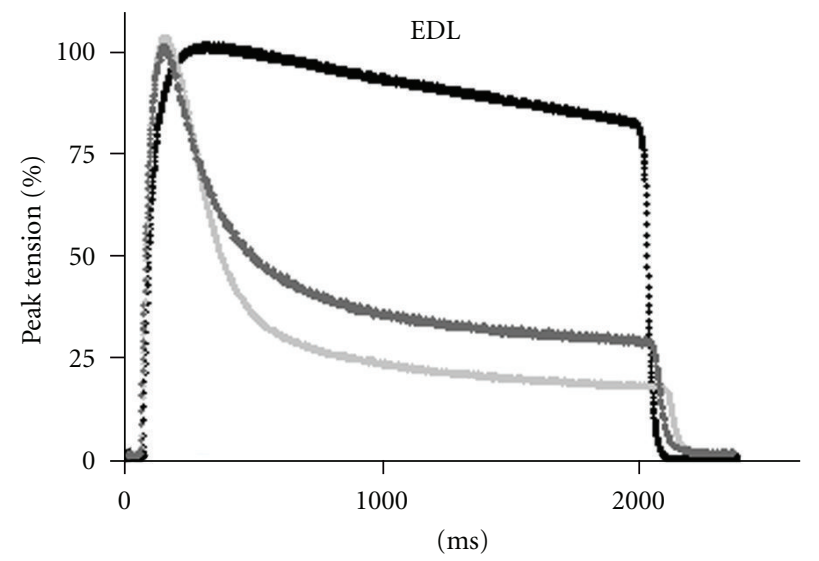

(a)

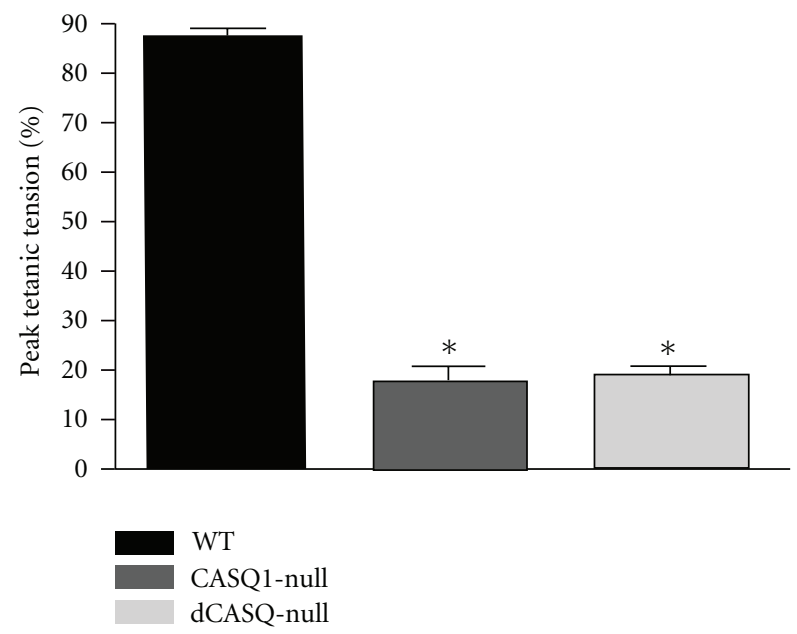

(b)

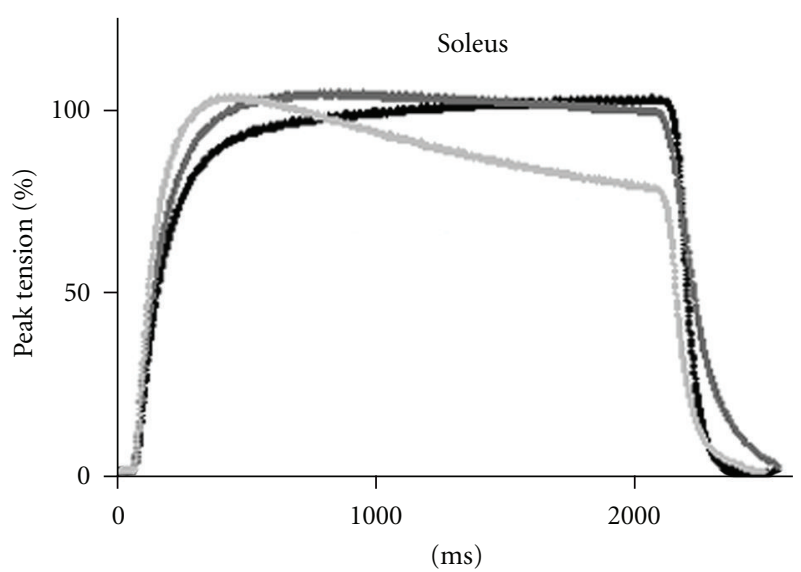

(c)

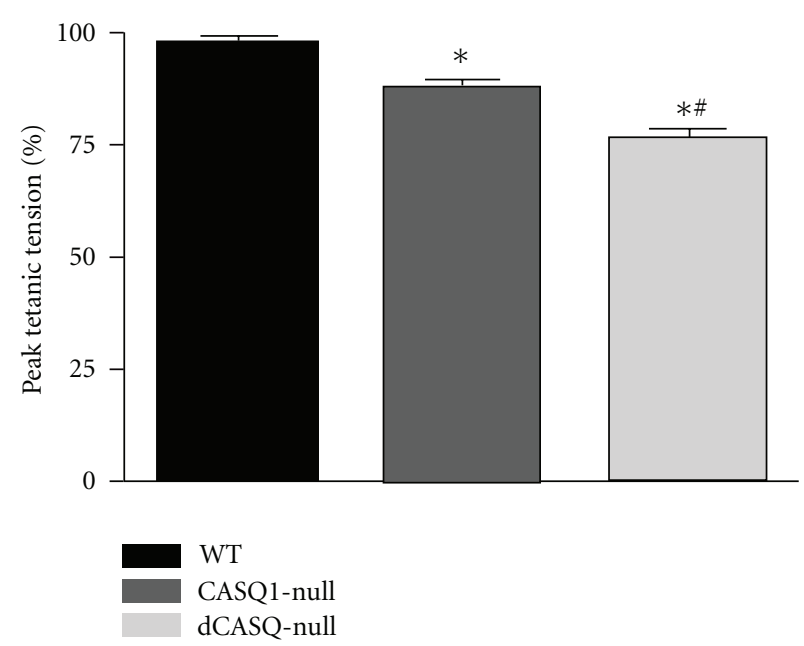

(d)

FIGURE 6: Tension decline and residual tension after 2 seconds of high-frequency stimulation in EDL and Soleus of CASQ1-null and dCASQnull mice. (a) and (b) When stimulated at high frequency $(2 \mathrm{~s}, 140 \mathrm{~Hz})$ EDL muscles of both CASQ1-null and dCASQ-null displayed a strong drop in tension (by about 80\%) compared to WT. (c) and (d) Conversely, in Soleus muscle (stimulated at $90 \mathrm{~Hz}$ for $2 \mathrm{~s}$ ) this effect was considerably less: compared to WT, tension was decreased by $\sim 15 \%$ in CASQ1-null and 25\% in dCASQ-null (EDL: WT $n=8$, CASQ1-null $n=10$, dCASQ null $n=9$, Soleus: WT $n=8$, CASQ1-null $n=10$, dCASQ null $n=10$ ). * Significantly different from WT; \#significantly different from CASQ1-null.

Soleus muscle in mice lacking both CASQ isoforms. The comparison of the structural and functional effect of the complete ablation of CASQ revealed two main distinctive features: (1) Soleus, but not EDL, shows a number of fibers with signs of structural degeneration (Figure 4); (2) EDL is unable to maintain active tension during a prolonged tetanus, while Soleus is only marginally affected (Figure 6). Both aspects require careful consideration.

4.1. Phenotype of dCASQ-Null Mice. Previous studies have shown that CASQ1-null mice are susceptible to spontaneous mortality and trigger $\mathrm{MH}$-like episodes when exposed to either heat or anesthesia [32, 34]. CASQ2-null mice display frequent episodes of catecholaminergic polymorphic ventricular tachycardia (CPVT), which can be triggered by a catecholamine challenge with the $\beta$-adrenergic agonist isoproterenol [31]. dCASQ-null mice are viable, but present high frequency of spontaneous death in male mice, even higher than that previously registered in CASQ1-null animals [32] (Figure 2). The specific reason for the increased spontaneous mortality rate of dCASQ-null mice is still under investigation, since CPVT in mice lacking CASQ2 is not lethal [31]. Grip strength test confirmed a significant impairment of the overall neuromuscular function (Figure 3(b)), similar to that of CASQ1-null mice. The lack of a significant difference in grip strength between CASQ1- and dCASQ-null mice probably reflects the fact that murine muscles show a great predominance of fast fibers expressing exclusively CASQ1 [2]. Finally, dCASQ-null mice show a reduced body weight which can be likely ascribed to a decrease in skeletal muscle mass due to a myopathic phenotype [35]. 
4.2. Early Onset of a Myopathy in Soleus Fibers: A Possible Explanation. We have recently reported that isolated muscles (EDL), muscle fibers (from FDBs), and myotubes lacking CASQ1 present elevated basal cytosolic $\mathrm{Ca}^{2+}$ at body temperature $[30,32]$. This abnormally high resting cytosolic $\mathrm{Ca}^{2+}$ causes abnormal development of muscle tension (contracture) when body temperature is raised above physiological values $\left(39-41^{\circ} \mathrm{C}\right)$ [32]. This reaction to heat explains why CASQ1-null mice are susceptible to trigger lethal malignant hyperthermia $(\mathrm{MH})$ like episodes when exposed to either high environmental temperatures or halogenated anesthetics [34]. In the present study we show that $\sim 30 \%$ of Soleus fibers from dCASQ-null mice present clear evidence of the early onset of a myopathic phenotype, which is not as evident in EDL fibers at the same age (4-6 months, Figure 4). These alterations, which resemble those described in other murine models of $\mathrm{MH}$ and central core disease (CCD) [36, 37], were not seen in Soleus fibers of CASQ1-null mice, suggesting that the abundant expression of CASQ2 in slow fibers (Figure 1) prevents the onset of the pathology. Preliminary data from our laboratory shows that also EDL of CASQ1-null mice (where a minor amount of CASQ2 is expressed) will eventually develop a similar myopathy with increasing age [38]. This considered, the difference between the two muscles is that Soleus develops a myopathy at an earlier stage. The reason can be tentatively found in the different capabilities of EDL and Soleus to deal with removal of $\mathrm{Ca}^{2+}$ from cytosol and with $\mathrm{SR} \mathrm{Ca}^{2+}$ leak.

In support to this view, it has been recently suggested that CASQ2 in slow muscle fibers may be important to prevent $\mathrm{Ca}^{2+}$ leakage [21]. The SR of slow fibers is saturated with $\mathrm{Ca}^{2+}$ at resting myoplasmic $\mathrm{Ca}^{2+}$ concentration, while the SR of fast fibers is only about one-third saturated with $\mathrm{Ca}^{2+}$ under equivalent conditions $[21,39]$. Such difference implies that the rate of SR $\mathrm{Ca}^{2+}$ uptake in fast fibers is predominantly controlled by myoplasmic $\mathrm{Ca}^{2+}$, while in slow fibers is more likely limited by the $\mathrm{Ca}^{2+}$ concentration within the SR lumen [39]. The intraluminal $\mathrm{Ca}^{2+}$ concentration is likely increased when the buffering action of CASQ is missing in the Soleus of dCASQ-null mice, causing serious challenge to reuptake. This would in turn result in excessive SR Ca ${ }^{2+}$ leak and $\left[\mathrm{Ca}^{2+}\right]_{\mathrm{i}}$, which will ultimately lead to the structural decay. The dramatic prolongation of the time to base (i.e., relaxation duration) of Soleus lacking both CASQ isoforms is in agreement with the impaired $\mathrm{Ca}^{2+}$ reuptake.

4.3. The Capability to Sustain Tetanic Tension Is Impaired in EDL, But Not in Soleus. The complete removal of CASQ in double-null mice decreases the ability of the SR to store $\mathrm{Ca}^{2+}$ both in fast and in slow muscle fibers. Slow Soleus fibers, but not fast EDL fibers, are able to sustain tension during a prolonged contractions (Figure 6), due to specific feature of the $\mathrm{Ca}^{2+}$ kinetics as discussed here below. The RYR-mediated $\mathrm{Ca}^{2+}$ release from the $\mathrm{SR}$ is approximately two times greater in fast compared to slow fibers. The larger size of CRUs and the higher density of RYR1 and DHPR in fast fibers is instrumental to this $[40,41]$. A greater $\mathrm{Ca}^{2+}$ release is needed in EDL fast fibers compared to Soleus slow fibers as the number of cytosolic $\mathrm{Ca}^{2+}$ binding sites is greater. In the first place, the troponin-C (TnC) isoform expressed in fast-twitch fibers presents two low affinity $\mathrm{Ca}^{2+}$ binding sites, whereas there is only one in the slowtwitch TnC isoform and, in addition, other cytosolic $\mathrm{Ca}^{2+}$ binding proteins must be saturated during the contractile cycle. Among them, there are parvalbumin, present at a concentration of $400-500 \mu \mathrm{mol} /$ liter in fast fibers but not in slow fibers [42] and containing two $\mathrm{Ca}^{2+}$ binding sites, and SERCA1, with a concentration of $120 \mu \mathrm{mol} /$ liter and two $\mathrm{Ca}^{2+}$ binding sites [40]. During a twitch (or the initial phase of a tetanus) the $\mathrm{Ca}^{2+}$ released can efficiently saturate $\mathrm{TnC}$ and SERCA and other minor $\mathrm{Ca}^{2+}$ buffers like Calmodulin, and the amount of 300-400 $\mu$ moles is likely to be sufficient, in agreement with published evidence [40, 43]. During a tetanic train of stimuli, more $\mathrm{Ca}^{2+}$ enters the cytosol through repeated releases, although the amplitude of the subsequent releases progressively decreases with the fifth release being only $10 \%$ of the first release $[40,43]$. In order to sustain tension for more than one second, it is necessary to saturate also parvalbumin, a process occurring with a slower kinetics related to calcium replacement for magnesium [44]: fast fibers deprived of CASQ fails to maintain a prolonged tetanic tension, probably because the $\mathrm{Ca}^{2+}$ released is not sufficient to saturate all cytosolic calcium-binding proteins, parvalbumin among them, with the result of reducing the $\mathrm{Ca}^{2+}$ available for $\mathrm{TnC}$, and therefore for tension generation. Conversely, a quite different scenario takes place in a typical slow fibers of Soleus where the lower SERCA density, the single low binding site of slow TnC, and, above all, the absence of parvalbumin [42] would prevent the tension decline observed in EDL. In this contest, very likely the minor reduction $(\sim 25 \%)$ in tetanic tension recorded in dCASQ-null Soleus (Figures 6(c) and 6(d)) could be almost completely ascribed to the presence of damaged fibers, which likely have compromised contractile function, and not to an insufficient amount of $\mathrm{Ca}^{2+}$ to activate contraction.

4.4. Conclusion Remarks. CASQ ablation has a differential effect in fast versus slow skeletal muscle fibers. Whereas in EDL CASQ is necessary to provide the large amount of $\mathrm{Ca}^{2+}$ required for a maximal sustained contraction, slowtwitch fibers are only moderately affected by the absence of CASQ during prolonged tetani. However, CASQ presence in Soleus seems necessary to help $\mathrm{Ca}^{2+}$ reuptake, reduce $\mathrm{Ca}^{2+}$ leakage, and control myoplasmic $\mathrm{Ca}^{2+}$, which otherwise will eventually lead to the early onset of a myopathy. In this aspect, slow fibers are reminiscent of cardiac myocytes where CASQ role is essential to control diastolic $\mathrm{Ca}^{2+}$ leakage [31]. These findings add novel information, which may help to better understand the differences in $\mathrm{Ca}^{2+}$ handling of fast and slow fibers and also offer new insights to unlock mechanisms leading to myopathies such as MH and CCD.

\section{Abbreviations Used in the Paper}

$\begin{array}{ll}\mathrm{Ca}^{2+}: & \text { Calcium ions } \\ \text { CASQ1 and CASQ2: } & \text { Skeletal and cardiac isoform of } \\ & \text { Calsequestrin } \\ \text { CCD: } & \text { Central core disease }\end{array}$




$\begin{array}{ll}\text { CRUs: } & \text { Calcium release units } \\ \text { DHPR: } & \text { Dihydropyridine receptors } \\ \text { EC Coupling: } & \text { Excitation-contraction coupling } \\ \text { EDL: } & \text { Extensor digitorum longus } \\ \text { EM: } & \text { Electron microscopy } \\ \text { MH: } & \text { Malignant hyperthermia } \\ \text { MHC: } & \text { Myosin heavy-chain } \\ \text { RYR1: } & \text { Ryanodine receptor type-1 } \\ \text { SERCA: } & \text { Sarcoendoplasmic reticulum } \mathrm{Ca}^{2+} \\ & \text { ATP-ase } \\ \text { SR: } & \text { Sarcoplasmic reticulum } \\ \text { TnC: } & \text { Troponin-C } \\ \text { T-Tubule: } & \text { Transverse tubule } \\ \text { WT: } & \text { Wild type. }\end{array}$

\section{Acknowledgments}

The authors thank Drs. P.D. Allen (Brigham \& Women's Hospital, Boston, MA) and B.C. Knollmann (Vanderbilt University, Nashville, TN) for providing the doubleCASQnull mouse colony used in the experiments. The authors also thank Dante Tatone, Cosmo Rossi, and Marco Dainese for technical assistance with equipment and mice housing. This study was supported by Research Grant no. GGP08153 from the Italian Telethon ONLUS Foundation to F. Protasi and C. Reggiani. Paolini and M. Quarta contributed equally to the work.

\section{References}

[1] S. Schiaffino and C. Reggiani, "Molecular diversity of myofibrillar proteins: gene regulation and functional significance," Physiological Reviews, vol. 76, no. 2, pp. 371-423, 1996.

[2] M. A. Pellegrino, M. Canepari, R. Rossi, G. D’Antona, C. Reggiani, and R. Bottinelli, "Orthologous myosin isoform and scaling of shortening velocity with body size in mouse, rat, rabbit and human muscles," Journal of Physiology, vol. 546, no. 3, pp. 677-689, 2003.

[3] C. Reggiani and T. T. Kronnie, "RyR isoforms and fibre type-specific expression of proteins controlling intracellular calcium concentration in skeletal muscles," Journal of Muscle Research and Cell Motility, vol. 27, no. 5-7, pp. 327-335, 2006.

[4] S. Carroll, P. Nicotera, and D. Pette, "Calcium transients in single fibers of low-frequency stimulated fast- twitch muscle of rat," American Journal of Physiology, vol. 277, no. 6, pp. C1122C1129, 1999.

[5] S. L. Carroll, M. G. Klein, and M. F. Schneider, "Decay of calcium transients after electrical stimulation in rat fast- and slow-twitch skeletal muscle fibres," Journal of Physiology, vol. 501, no. 3, pp. 573-588, 1997.

[6] O. Delbono, K. S. O'Rourke, and W. H. Ettinger, "Excitationcalcium release uncoupling in aged single human skeletal muscle fibers," Journal of Membrane Biology, vol. 148, no. 3, pp. 211-222, 1995.

[7] M. W. Fryer and I. R. Neering, "Actions of caffeine on fast- and slow-twitch muscles of the rat," Journal of Physiology, vol. 416, pp. 435-454, 1989.

[8] C. Franzini-Armstrong, "Studies of the triad. 3. Structure of the junction in fast twitch fibers," Tissue and Cell, vol. 4, no. 3, pp. 469-478, 1972.
[9] C. Franzini Armstrong, L. Landmesser, and G. Pilar, "Size and shape of transverse tubule openings in frog twitch muscle fibers," Journal of Cell Biology, vol. 64, no. 2, pp. 493-497, 1975.

[10] C. Paolini, J. D. Fessenden, I. N. Pessah, and C. FranziniArmstrong, "Evidence for conformational coupling between two calcium channels," Proceedings of the National Academy of Sciences of the United States of America, vol. 101, no. 34, pp. 12748-12752, 2004.

[11] F. Protasi, C. Franzini-Armstrong, and P. D. Allen, "Role of ryanodine receptors in the assembly of calcium release units in skeletal muscle," Journal of Cell Biology, vol. 140, no. 4, pp. 831-842, 1998.

[12] E. Rios, J. Ma, and A. Gonzalez, "The mechanical hypothesis of excitation-contraction (EC) coupling in skeletal muscle," Journal of Muscle Research and Cell Motility, vol. 12, no. 2, pp. 127-135, 1991.

[13] M. F. Schneider, "Control of calcium release in functioning skeletal muscle fibers," Annual Review of Physiology, vol. 56, pp. 463-484, 1994.

[14] K. P. Campbell, D. H. MacLennan, A. O. Jorgensen, and M. C. Mintzer, "Purification and characterization of calsequestrin from canine cardiac sarcoplasmic reticulum and identification of the 53,000 dalton glycoprotein," Journal of Biological Chemistry, vol. 258, no. 2, pp. 1197-1204, 1983.

[15] D. H. MacLennan, V. Shoshan, and D. S. Wood, "Studies of $\mathrm{Ca} 2+$ release from sarcoplasmic reticulum," Annals of the New York Academy of Sciences, vol. 402, pp. 470-477, 1982.

[16] C. Franzini-Armstrong, L. J. Kenney, and E. VarrianoMarston, "The structure of calsequestrin in triads of vertebrate skeletal muscle: a deep-etch study," Journal of Cell Biology, vol. 105, no. 1, pp. 49-56, 1987.

[17] A. O. Jorgensen, A. C. Y. Shen, K. P. Campbell, and D. H. MacLennan, "Ultrastructural localization of calsequestrin in rat skeletal muscle by immunoferritin labeling of ultrathin frozen sections," Journal of Cell Biology, vol. 97, no. 5 I, pp. 1573-1581, 1983.

[18] E. Damiani, G. Tobaldin, P. Volpe, and A. Margreth, "Quantitation of ryanodine receptor of rabbit skeletal muscle, heart and brain," Biochemical and Biophysical Research Communications, vol. 175, no. 3, pp. 858-865, 1991.

[19] R. Sacchetto, P. Volpe, E. Damiani, and A. Margreth, "Postnatal development of rabbit fast-twitch skeletal muscle: accumulation, isoform transition and fibre distribution of calsequestrin," Journal of Muscle Research and Cell Motility, vol. 14, no. 6, pp. 646-653, 1993.

[20] E. Leberer, K. T. Hartner, and D. Pette, "Postnatal development of Ca2+-sequestration by the sarcoplasmic reticulum of fast and slow muscles in normal and dystrophic mice," European Journal of Biochemistry, vol. 174, no. 2, pp. 247-253, 1988.

[21] R. M. Murphy, N. T. Larkins, J. P. Mollica, N. A. Beard, and G. D. Lamb, "Calsequestrin content and SERCA determine normal and maximal $\mathrm{Ca} 2+$ storage levels in sarcoplasmic reticulum of fast- and slow-twitch fibres of rat," Journal of Physiology, vol. 587, no. 2, pp. 443-460, 2009.

[22] H. Park, Y. I. Park, E. Kim et al., "Comparing skeletal and cardiac calsequestrin structures and their calcium binding: a proposed mechanism for coupled calcium binding and protein polymerization," Journal of Biological Chemistry, vol. 279, no. 17, pp. 18026-18033, 2004.

[23] N. A. Beard, D. R. Laver, and A. F. Dulhunty, "Calsequestrin and the calcium release channel of skeletal and cardiac muscle," Progress in Biophysics and Molecular Biology, vol. 85, no. 1, pp. 33-69, 2004. 
[24] N. A. Beard, M. M. Sakowska, A. F. Dulhunty, and D. R. Laver, "Calsequestrin is an inhibitor of skeletal muscle ryanodine receptor calcium release channels," Biophysical Journal, vol. 82, no. 1, pp. 310-320, 2002.

[25] N. Ikemoto, M. Ronjat, L. G. Mészáros, and M. Koshita, "Postulated role of calsequestrin in the regulation of calcium release from sarcoplasmic reticulum," Biochemistry, vol. 28, no. 16, pp. 6764-6771, 1989.

[26] N. A. Beard, L. Wei, and A. F. Dulhunty, "Ca2+ signaling in striated muscle: the elusive roles of triadin, junctin, and calsequestrin," European Biophysics Journal, vol. 39, no. 1, pp. 27-36, 2009.

[27] N. Chopra, P. J. Kannankeril, T. Yang et al., "Modest reductions of cardiac calsequestrin increase sarcoplasmic reticulum Ca2+ leak independent of luminal $\mathrm{Ca} 2+$ and trigger ventricular arrhythmias in mice," Circulation Research, vol. 101, no. 6, pp. 617-626, 2007.

[28] C. Paolini, M. Quarta, A. Nori et al., "Reorganized stores and impaired calcium handling in skeletal muscle of mice lacking calsequestrin-1," Journal of Physiology, vol. 583, no. 2, pp. 767784, 2007.

[29] L. Royer, M. Sztretye, C. Manno et al., "Paradoxical buffering of calcium by calsequestrin demonstrated for the calcium store of skeletal muscle," Journal of General Physiology, vol. 136, no. 3, pp. 325-338, 2010.

[30] M. Canato, M. Scorzeto, M. Giacomello, F. Protasi, C. Reggiani, and G. J. M. Stienen, "Massive alterations of sarcoplasmic reticulum free calcium in skeletal muscle fibers lacking calsequestrin revealed by a genetically encoded probe," Proceedings of the National Academy of Sciences of the United States of America, vol. 107, no. 51, pp. 22326-22331, 2010.

[31] B. C. Knollmann, N. Chopra, T. Hlaing et al., "Casq2 deletion causes sarcoplasmic reticulum volume increase, premature $\mathrm{Ca} 2+$ release, and catecholaminergic polymorphic ventricular tachycardia," Journal of Clinical Investigation, vol. 116, no. 9, pp. 2510-2520, 2006.

[32] M. Dainese, M. Quarta, A. D. Lyfenko et al., "Anestheticand heat-induced sudden death in calsequestrin-1-knockout mice," FASEB Journal, vol. 23, no. 6, pp. 1710-1720, 2009.

[33] A. M. Connolly, R. M. Keeling, S. Metha, A. Pestronk, and J. R. Sanes, "Three mouse models of muscular dystrophy: the natural history of strength and fatigue in dystrophindystrophin/utrophin-, and laminin alpha2-deficient mice," Neuromuscular Disorders, vol. 11, pp. 703-712, 2001.

[34] F. Protasi, C. Paolini, and M. Dainese, "Calsequestrin-1: a new candidate gene for malignant hyperthermia and exertional/environmental heat stroke," Journal of Physiology, vol. 587, no. 13, pp. 3095-3100, 2009.

[35] C. Paolini, M. Quarta, A. Nori et al., "Lack of Calsequestrin1 causes impaired maturation and a progressive myopathy in skeletal fibers," Journal of Muscle Research and Cell Motility, vol. 30, p. A322, 2009.

[36] S. Boncompagni, R. E. Loy, R. T. Dirksen, and C. FranziniArmstrong, "The I4895T mutation in the type 1 ryanodine receptor induces fiber-type specific alterations in skeletal muscle that mimic premature aging," Aging Cell, vol. 9, no. 6, pp. 958-970, 2010.

[37] S. Boncompagni, A. E. Rossi, M. Micaroni et al., "Characterization and temporal development of cores in a mouse model of malignant hyperthermia," Proceedings of the National Academy of Sciences of the United States of America, vol. 106, no. 51, pp. 21996-22001, 2009.
[38] C. Paolini, M. Quarta, M. Dainese et al., "Initial characterization of CASQ1/CASQ2 knockout (double CASQ-null) mice," Biophysical Journal, vol. 98, no. 3, supplement 1, pp. 546a547a, 2010.

[39] M. W. Fryer and D. G. Stephenson, "Total and sarcoplasmic reticulum calcium contents of skinned fibres from rat skeletal muscle," Journal of Physiology, vol. 493, no. 2, pp. 357-370, 1996.

[40] S. M. Baylor and S. Hollingworth, "Sarcoplasmic reticulum calcium release compared in slow-twitch and fast-twitch fibres of mouse muscle," Journal of Physiology, vol. 551, no. 1, pp. 125-138, 2003.

[41] C. Franzini-Armstrong, F. Protasi, and V. Ramesh, "Shape, size, and distribution of $\mathrm{Ca} 2+$ release units and couplons in skeletal and cardiac muscles," Biophysical Journal, vol. 77, no. 3, pp. 1528-1539, 1999.

[42] M. R. Celio and C. W. Heizmann, "Calcium-binding protein parvalbumin is associated with fast contracting muscle fibres," Nature, vol. 297, no. 5866, pp. 504-506, 1982.

[43] G. S. Posterino and G. D. Lamb, "Effect of sarcoplasmic reticulum $\mathrm{Ca} 2+$ content on action potential-induced $\mathrm{Ca} 2+$ release in rat skeletal muscle fibres," Journal of Physiology, vol. 551, no. 1, pp. 219-237, 2003.

[44] T. T. Hou, J. D. Johnson, and J. A. Rall, "Parvalbumin content and $\mathrm{Ca} 2+$ and $\mathrm{Mg} 2+$ dissociation rates correlated with changes in relaxation rate of frog muscle fibres," Journal of Physiology, vol. 441, pp. 285-304, 1991. 

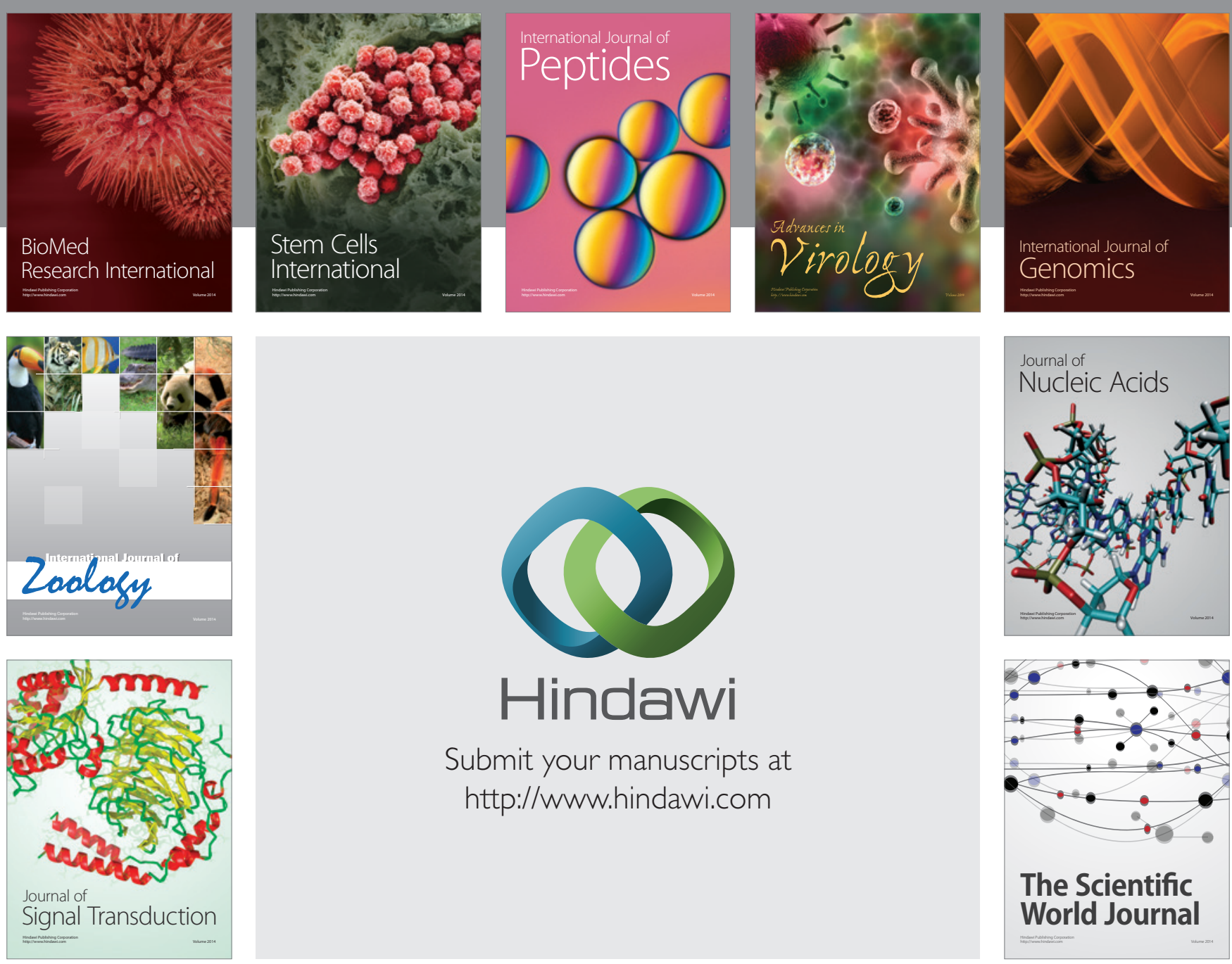

Submit your manuscripts at

http://www.hindawi.com
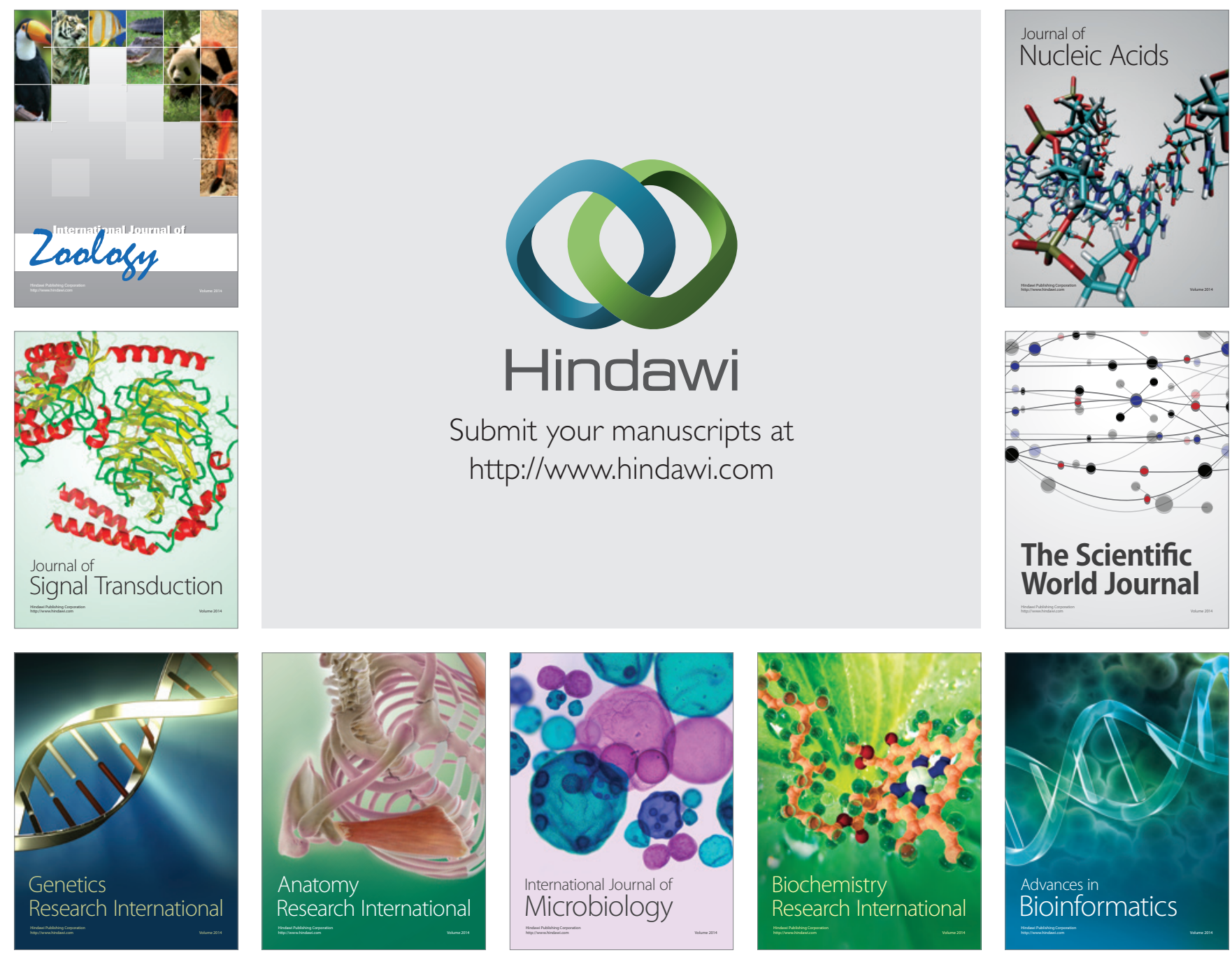

The Scientific World Journal
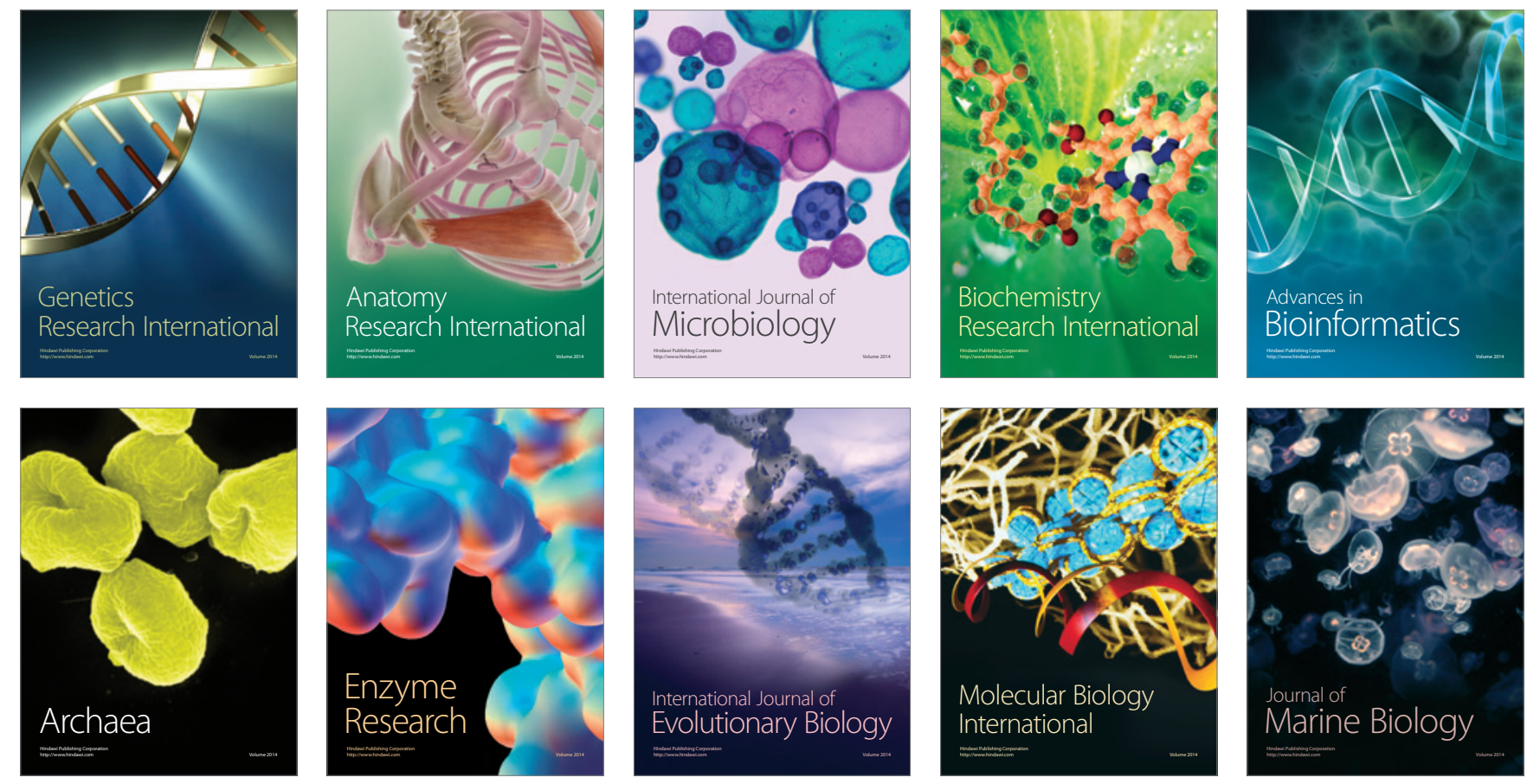